\title{
November 2017 Imaging Case of the Month
}

\author{
Michael B. Gotway, MD' \\ Isabel Mira-Avendano, MD2 \\ ${ }^{1}$ Mayo Clinic Arizona, Scottsdale AZ USA \\ ${ }^{2}$ Mayo Clinic Jacksonville, FL USA
}

Clinical History: A 70-year-old white woman with a remote history of smoking and mild gastroesophageal reflux disease presented with complaints of a dry cough and shortness of breath, present for some time but worsening over the previous 8 months. No hemoptysis was noted and the patient did not complain of chest pain. No history of syncope was noted.

Physical examination was largely unremarkable and the patient's oxygen saturation was $86 \%$ on room air, $90 \%$ on $4 \mathrm{~L} / \mathrm{m}$ by mask. The patient's vital signs were within normal limits.

Laboratory evaluation was unremarkable. Quantiferon testing for Mycobacterium tuberculosis was negative, and testing for coccidioidomycosis was unrevealing. Enhanced thoracic CT (Figure 1) was performed.

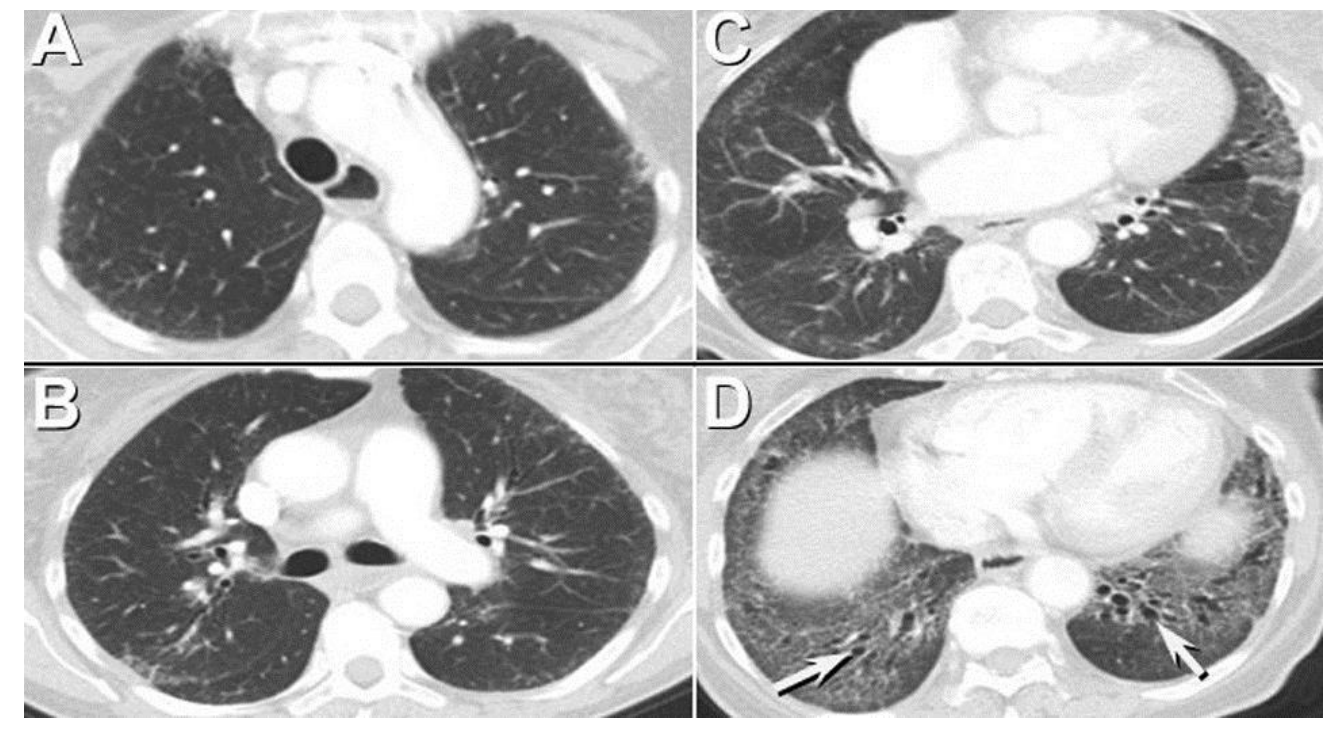

Figure 1. Representative images from the thoracic CT scan in lung windows.

Which of the following statements regarding the thoracic CT is most accurate?

1. The thoracic CT shows advanced destructive emphysema

2. The thoracic CT shows bilateral, basal and subpleural predominant reticulation associated with ground-glass opacity, architectural distortion, and traction bronchiectasis

3. The thoracic CT shows multifocal lobular consolidation

4. The thoracic CT shows multifocal small pulmonary cysts

5. The thoracic CT shows small cavitary pulmonary nodules 


\section{Correct! \\ 2. The thoracic CT shows bilateral, basal and subpleural predominant reticulation associated with ground-glass opacity, architectural distortion, and traction bronchiectasis}

The thoracic CT shows bilateral, basal and subpleural predominant reticulation associated with ground-glass opacity, architectural distortion, and traction

bronchiectasis, characteristic of fibrotic lung disease. There is no evidence of cystic lung disease, emphysema, or honeycombing. No significant lobular consolidation is present.

Which of the following represents the most appropriate description for the thoracic CT pattern present?

1. "Crazy - paving"

2. A fibrotic non-specific interstitial pneumonia pattern

3. A lymphocytic interstitial pneumonia pattern

4. A usual interstitial pneumonia pattern

5. An organizing pneumonia pattern 


\section{Correct! \\ 2. A fibrotic non-specific interstitial pneumonia pattern}

The bilateral, basal and subpleural predominant reticulation associated with groundglass opacity, architectural distortion, and traction bronchiectasis seen on the thoracic CT represents a fibrotic non-specific interstitial pneumonia (NSIP) pattern. This pattern can also be seen with "early" usual interstitial pneumonia (UIP), although the UIP pattern is considered to be present when honeycomb cysts are seen in the context of the aforementioned findings. The "organizing pneumonia" (OP) pattern consists of multifocal, typically bilateral, areas of peripheral, often frankly subpleural, consolidation, possibly with areas of ground-glass opacity, often also showing a peribronchial distribution as well. Occasionally the CT findings may be migratory and/or recurrent in patients with OP. Recognized variations of the OP pattern include areas of perilobular consolidation, the "reverse ground-glass halo" sign (also referred to as the "atoll" sign), and solitary or multiple nodules that may show air bronchograms. Rarely OP may present as reticular abnormalities with architectural distortion and traction bronchiectasis at thoracic CT and may closely resemble the fibrotic NSIP pattern. A number of thoracic CT appearances for lymphocytic interstitial pneumonia (LIP) have been described, and there is no specifically recognized "LIP pattern" in the same sense as described for NSIP, UIP, and OP. The main thoracic CT patterns described in patients with LIP include areas of ground-glass opacity and ground-glass opacity centrilobular nodules, possibly with interlobular septal thickening, perilymphatic nodules, and multiple randomly distributed thin-walled cysts; the latter pattern is often discussed as the most "recognizable" thoracic CT pattern of LIP, although the appearance is ultimately non-specific and can be closely mimicked by lymphangioleiomyomatosis, BirtHogg-Dubé syndrome, and various causes of small airway obstruction. The "crazypaving" pattern at thoracic CT represents "thickened interlobular septa and intralobular lines superimposed on a background of ground-glass opacity, resembling irregularly shaped paving stones. The crazy-paving pattern is often sharply demarcated from more normal lung and may have a geographic outline." This pattern has been classically associated with pulmonary alveolar proteinosis, but is not completely specific for that disorder and has been described in a number of conditions.

Which of the following represents the most appropriate next step for the management of this patient?

1. Anti-nuclear antibody and Scl-70 antibody testing

2. Endoscopic ultrasound

3. Refer for surgical lung biopsy

4. Repeat CT using high-resolution technique

5. Thoracic magnetic resonance angiogram (MRA) 


\section{Correct! \\ 1. Anti-nuclear antibody and Scl-70 antibody testing}

The NSIP pattern can be seen idiopathically and can be very difficult to differentiate from "early" UIP. In fact, many patients with the thoracic CT interpretation of "possible" UIP using to the American Thoracic Society (ATS) consensus criteria, particularly when the only CT feature lacking for an interpretation of "definite UIP" at thoracic CT is the absence of honeycombing, will be shown to have UIP on surgical biopsy. However, when the NSIP pattern is present on thoracic CT, systemic disorders associated with this pattern should be sought, most notably connective tissue disorders. Inasmuch as possible, the presence of a connective tissue disorder should be excluded before obtaining a surgical biopsy in patients with interstitial lung disease, as the histopathological pattern of lung involvement in patients with connective tissue disorders rarely influences treatment. Therefore, surgical lung biopsy is premature at this point. A repeat thoracic CT using high-resolution technique (HRCT; which implies the use of narrow section width, prone inspiratory imaging, and post-expiratory imaging) can be useful for the assessment of patients with interstitial lung diseases; in particular, prone imaging often proves valuable to determine if the basal lung abnormalities- typical of many interstitial lung disorders, particularly several of the idiopathic interstitial pneumonias- persists on prone imaging, as opposed to resolving on prone imaging, as is typical of reversible basal lung opacity/atelectasis. However, in this patient, the basal lung findings are quite pronounced and are unlikely to reflect reversible basal lung opacity / atelectasis, so repeat HRCT is not required. Endoscopic ultrasound would not be of value for this patient at this point- no target appropriate for biopsy is present. Thoracic MRA could prove useful to assess for vasculitis but would not add useful information to that already obtained with the thoracic CT.

Both the anti-nuclear antibody and Scl-70 antibody tests were positive. Pulmonary function testing showed a forced vital capacity (FVC) of $67 \%$ predicted, forced expiratory volume in 1 second $\left(\mathrm{FEV}_{1}\right) 62 \%$ predicted, total lung capacity (TLC) $64 \%$ predicted, and diffusing capacity for carbon monoxide (DLCO) of $20 \%$ predicted. She was capable of 216 meters during her 6-minute walk test. The patient was presumptively started on pirfenidone.

Which of the following statements is the most appropriate next step for the management of this patient?

1. Bronchoscopy with cryobiopsy

2. Continue pirfenidone therapy for possible usual interstitial pneumonia

3. Discontinue pirfenidone therapy and consult rheumatology

4. Obtain ${ }^{133} \mathrm{Xe}$-Ventilation - ${ }^{99 m}$ Tc-perfusion scintigraphy

5. Right heart catheterization 


\section{Correct! \\ 3. Discontinue pirfenidone therapy and consult rheumatology}

Pirfenidone therapy has recognized benefits for reducing lung function decline in patients with usual interstitial pneumonia/idiopathic pulmonary fibrosis, but that diagnosis has not been established for this patient and therefore this therapy is not appropriate for this patient. The presence of an NSIP pattern at thoracic CT and the positive antibody testing raises concern for a connective tissue disorder, and therefore, consultation with rheumatology is appropriate. Right heart catheterization could be considered given the relatively disproportionately low DLCO compared with the FVC, but typically the investigation of pulmonary hypertension would start non-invasively with echocardiography. Bronchoscopy may provide useful information in the evaluation of patients with diffuse lung disease, occasionally providing the correct diagnosis, or at least excluding some competing causes, particularly infections, that would be inappropriately treated should immunosuppressive therapy be employed. Cryobiopsy may provide tissue for histopathological sampling and could allow a definitive diagnosis for the fibrotic lung disease in this patient, but the patient's clinical presentation and thoracic CT findings raise the possibility of a systemic disorder rather than an idiopathic interstitial pneumonia, and it is therefore unlikely that lung tissue sampling would provide information that will alter or substantially impact therapy. ${ }^{133}$ Xe-Ventilation - ${ }^{99 m}$ Tc-perfusion scintigraphy would be useful to assess for acute or chronic thromboembolic disease or possibly to assess for relative pulmonary perfusion prior to thoracic surgery, but these considerations or probably not appropriate for this patient. The reduced DLCO does raise concern for pulmonary vascular disease, but the previous thoracic CT was an enhanced study and showed no evidence of acute or chronic pulmonary emboli. It would be highly unlikely that ${ }^{133} \mathrm{Xe}$-Ventilation - ${ }^{99 \mathrm{~m}}$ Tcperfusion scintigraphy would reveal high-probability results in the context of underlying structural lung disease with a recent enhanced thoracic CT examination showing no evidence of pulmonary emboli.

Upon further questioning and examination, the patient did note intermittent finger swelling for the previous 2 years and had intermittent symptoms consistent with Raynaud's phenomenon. No skin ulceration was present, but skin thickening and hardening over the fingers and hands, as well as subcutaneous tissue atrophy, was present, consistent with sclerodactyly.

Which of the following represents the most likely diagnosis for this patient?

1. Hypertrophic osteoarthropathy

2. Macrodactyly

3. Systemic sclerosis

4. Thyroiditis

5. Yellow nail syndrome 


\section{Correct! \\ 3. Systemic sclerosis}

Hypertrophic osteoathropathy is one of a number of conditions that can affect the digits in the form of digital clubbing, but the description of the patient's finger and hand findings is not consistent with this disorder. The yellow nail syndrome consists of the combination of yellow dystrophic nails, peripheral lymphedema and bronchiectasis with recurrent lower respiratory tract infections. This disorder is quite rare, and the physical examination findings are not consistent with this disorder. Furthermore, the basal bronchiectasis at thoracic CT in this patient is due to fibrotic disease (traction bronchiectasis), not intrinsic destruction of the airway walls resulting in bronchial dilation. Macrodactyly, or focal gigantism, represents enlargement of one or more digits, often the fingers, due to excessive growth of the underlying bone and soft tissue. This disorder is typically congenital in nature, often occurring in the context of neurofibromatosis, vascular malformations, multiple enchondromatosis, or even tuberous sclerosis, but does not fit with the patient's physical examination findings. Finally, autoimmune thyroiditis may cause thyroid acropachy, which presents as digital swelling, clubbing, and periostitis, typically in the context of the ophthalmopathy and dermopathy associated with thyroid diseases- this patient does not have those stigmata and the physical examination findings do not fit with thyroid acropachy. However, the patient's physical examination findings, thoracic CT findings, and antibody profile fit well with systemic sclerosis.

The patient was diagnosed with systemic sclerosis and pirfenidone was discontinued and mycophenolate mofetil and oxygen were started. Transthoracic echocardiography was performed, which showed normal left ventricular size and function and an estimated mean pulmonary arterial pressure of $43 \mathrm{mmHg}$ with an estimated right ventricular systolic pressure of $63 \mathrm{mmHg}$. The right ventricular size and function were thought to be normal.

Which of the following statements represents the most appropriate next step for the management of this patient?

1. Cardiac MRI

2. Continue oxygen and mycophenolate mofetil therapy and reassess in 3 months

3. Right heart catheterization

4. Surgical lung biopsy

5. Transesophageal echocardiography 


\section{Correct! \\ 3. Right heart catheterization}

Given the clinical context and the transthoracic echocardiographic findings, pulmonary hypertension is likely, but right heart catheterization is required to confirm this diagnosis, and that diagnosis should be established or excluded sooner rather than later. A repeat transesophageal echocardiogram could be of benefit if the findings at transthoracic echocardiography were unclear, the transthoracic echocardiogram was technically limited, or an intracardiac shunt was suspected, but these considerations are not relevant to this patient. As noted previously, in the context of systemic sclerosis, the pattern of lung injury generally does not impact the course of therapy, and therefore tissue sampling is not required and may result in a greater rate of complication given the possible pulmonary hypertension. Cardiac MRI may be of benefit in patients with various cardiac diseases, including the possibility of pulmonary hypertension, and probably provides the most accurate method to assess right ventricular function and is particularly valuable when echocardiographic examinations are technically limited. Cardiac MR can provide data suggesting the presence of, and possible cause for, pulmonary hypertension, but this examination is not a substitute for the right heart catheterization confirmation of pulmonary hypertension.

Right heart catheterization showed a mean pulmonary arterial pressure of $34 \mathrm{mmHg}$, and pulmonary capillary wedge pressure of $8 \mathrm{mmHg}$, and a pulmonary vascular resistance of 4.8 Wood units. The patient was continued on mycophenolate mofetil and oxygen therapy and did well for three months, after which she presented with worsening dyspnea, now at rest, new lower extremity edema, and worsening hypoxemia.

Enhanced thoracic CT (Figure 2) was performed.

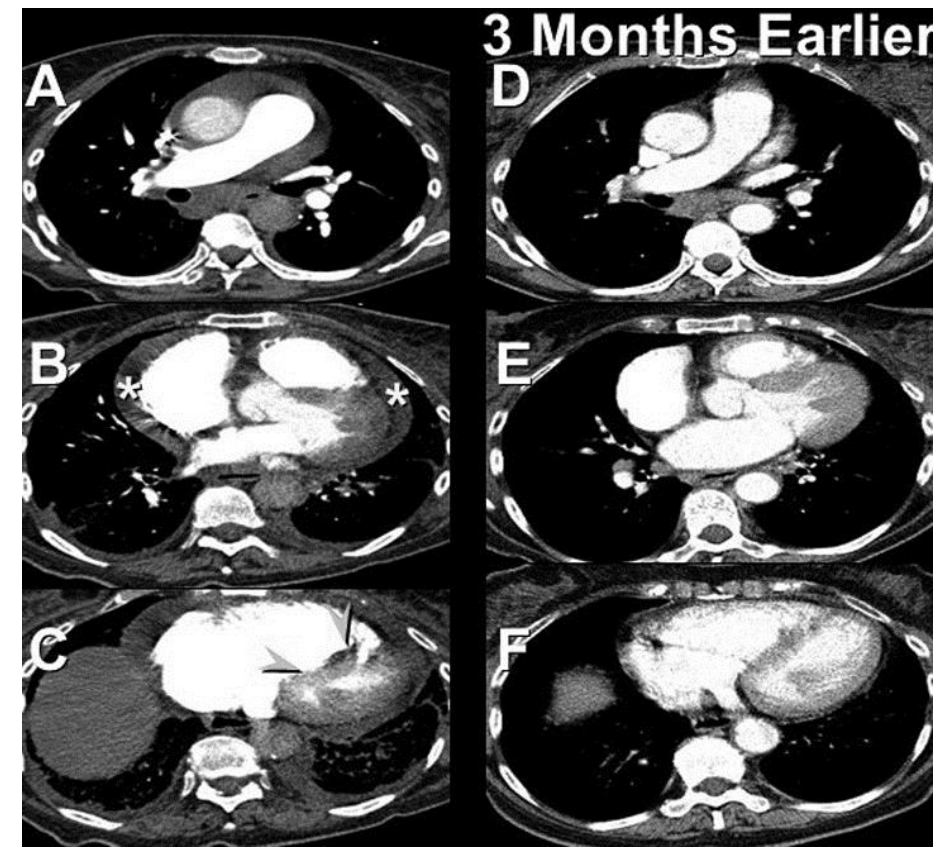

Figure 2. Contrast-enhanced pulmonary CT angiography. 
Which of the following represents the most likely diagnosis for this patient?

1. Acute exacerbation of interstitial pneumonia

2. Acute pulmonary emboli

3. Cor pulmonale

4. Increased pressure edema

5. Pulmonary arterial dissection 


\section{Correct! \\ 3. Cor pulmonale}

The thoracic CT, performed as a CT pulmonary angiogram, shows the pre-exiting fibrotic lung disease to be stable, without new infiltrative abnormalities, but with new pericardial effusion, enlargement of the right atrium and right ventricle with leftward bowing of the interventricular septum; the latter findings are consistent with acutely worsening pulmonary hypertension and right ventricular failure. The absence of new ground-glass opacity and / or consolidation superimposed on the fibrotic lung disease, excludes an acute exacerbation of the fibrotic lung disease. The left ventricle does not appear enlarged at CT and no interlobular septal thickening is evident, which argues against increased pressure edema. Pulmonary arterial dissection is a rare entity that is most commonly encountered in the context of pulmonary hypertension, but the characteristic appearance of pulmonary arterial dissection- a linear "intimal flap" within the pulmonary artery, is not present.

The patient underwent repeat transthoracic echocardiography which showed an estimated right ventricular systolic pressure of $115 \mathrm{mmHg}$, an estimated mean pulmonary arterial pressure of $78 \mathrm{mmHg}$, and severe right ventricular enlargement and dysfunction with a "D-shaped" right ventricle and a moderate pericardial effusion. The patient's oxygen saturation was $76 \%$ on room air, increasing to $91 \%$ by mask. Repeat right heart catheterization showed a mean pulmonary arterial pressure of $62 \mathrm{mmHg}$, pulmonary capillary wedge pressure of $32 \mathrm{mmHg}$ (thought to be attributable to impaired left ventricular filling owing to leftward bowing of the interventricular septum and marked enlargement of the right ventricle), and a pulmonary vascular resistance of 12 Wood units. The patient was treated with aggressive diuresis, maintained on mycophenolate mofetil and oxygen therapy, and inhaled prostacyclin therapy was started, with a good response. After three months, repeat transthoracic echocardiography showed reduction in the now-mild right ventricular enlargement and return of a normal configuration of the interventricular septum, with right ventricular end-systolic pressure decreased to 63 $\mathrm{mmHg}$.

Diagnosis: Interstitial lung disease associated with systemic sclerosis with development of pulmonary hypertension

\section{References}

1. Hansell DM, Bankier AA, MacMahon H, McLoud TC, Müller NL, Remy J. Fleischner Society: glossary of terms for thoracic imaging. Radiology. 2008; 246(3):697-722. [CrossRef] [PubMed]

2. Austin JH, Müller NL, Friedman PJ, Hansell DM, Naidich DP, Remy-Jardin M, Webb WR, Zerhouni EA. Glossary of terms for CT of the lungs: recommendations of the Nomenclature Committee of the Fleischner Society. Radiology. 1996; 200(2):327-31. [CrossRef] [PubMed]

3. Rowan C, Hansell DM, Renzoni E, Maher TM, Wells AU, Polkey MI, Rehal PK, Ibrahim WH, Kwong GN, Colby TV, Pistolesi M, Bigazzi F, Comin CE, Nicholson AG. 
Diffuse cystic lung disease of unexplained cause with coexistent small airway disease: a possible causal relationship? Am J Surg Pathol. 2012;36(2):228-34. [CrossRef] [PubMed]

4. Kawano-Dourado L, Baldi BG, Dias OM, Bernardi FD, Carvalho CR, Dolhnikoff $M$, Kairalla RA. Scattered lung cysts as the main radiographic finding of constrictive bronchiolitis. Am J Respir Crit Care Med. 2012;186(3):294-5. [CrossRef] [PubMed]

5. Johkoh T, Itoh H, Müller NL, Ichikado K, Nakamura H, Ikezoe J, Akira M, Nagareda T. Crazy-paving appearance at thin-section CT: spectrum of disease and pathologic findings. Radiology. 1999;211(1):155-60. [CrossRef] [PubMed]

6. Raghu G, Collard HR, Egan JJ, et. al., ATS/ERS/JRS/ALAT committee in IPF: an official ATS/ERS/JRS/ALAT statement: IPF evidence-based guidelines for diagnosis \& management. Am J Respir Crit Care Med. 2011;183:788-824. [CrossRef] [PubMed]

7. Henry TS, Little BP, Veeraraghavan S, Bhalla S, and Elicker BM. The spectrum interstitial lung disease in connective tissue disease. J Thorac Imag. 2016;31:65-77. [CrossRef] [PubMed]

8. Capobianco J, Grimberg A, Thompson BM, Antunes VB, Jasinowodolinski D, Meirelles GS. Thoracic manifestations of collagen vascular diseases. Radiographics. 2012; 32(1):33-50. [CrossRef] [PubMed] 